\title{
Pediatric inflammatory multisystem syndrome temporally associated with COVID-19 - a novel condition in children and adolescents
}

\author{
Małgorzata Wieteska' ${ }^{1}$, Dominik Maj², Paweł Stanicki ${ }^{1}$ \\ 'Department of Epidemiology and Clinical Research Methodology, Medical University of Lublin, Poland \\ ${ }^{2} 1^{\text {st }}$ Chair and Department of Cardiology, Medical University of Warsaw, Poland
}

\section{ABSTRACT}

In March 2020, World Health Organization (WHO) declared the outbreak of the coronavirus disease 2019 (COVID-19) a pandemic. Pediatric inflammatory multisystem syndrome temporally associated with COVID-19 (PIMS-TS) also known as multisystem inflammatory syndrome in children (MIS-C) is a new phenomenon highly correlated with COVID-19. Recent research suggests that this new disease in children and adolescents is associated with severe acute respiratory syndrome coronavirus 2 (SARS-CoV-2) infection. It is a generalized inflammatory reaction suggestive of a likely autoimmune etiology. Symptoms of PIMS-TS are diverse and wide ranging. The disorder develops in children and adolescents as a result of dysregulation of the immune system, but the exact pathogenesis of PIMS-TS remains unknown. Consequently, the most effective types of therapy include supportive care, symptomatic and immunomodulating treatment. The ongoing international study of this condition may provide information on the treatment options, clinical trials and management process.

KEY WORDS:

COVID-19, MIS-C, PIMS-TS, SARS-CoV-2.

\section{INTRODUCTION AND OBJECTIVE}

In December 2019, the first case of a novel contagious disease, named coronavirus disease 2019 (COVID-19) was identified in Hubei, a province of China. The disease has spread worldwide within a short period of time. COVID-19 has therefore become a serious and immediate challenge for public healthcare [1]. COVID-19 has been confirmed in more than 100 million people throughout the world by January 2021 [2]. Moreover, the case-fatality rate is in the range of 1 to $3 \%$. Advanced age and signs of metabolic syndrome represent negative prognostic factors in patients with COVID-19 [3]. An overwhelming spread of the COVID-19 pandemic led to many long term implications such as cardiopulmo- nary, glucometabolic and neuropsychiatric complications or social disruption $[1,4]$. The clinical spectrum appears varied, although a severe course of the infection affects 1 out of 10 patients. Dyspnea, hypoxemia and extensive pulmonary consolidations associated with pneumonia indicate a serious clinical status of patient. Studies suggest that clinical manifestations of COVID-19 are generally milder in children compared with adults. According to the research $79 \%$ of children with COVID-19 confirmed by reverse transcriptase-polymerase-chain-reaction (RT-PCR) testing are asymptomatic or have mild disease. Most common symptoms in pediatric patients are cough (48\%) and fever (47\%). Slight dyspnea, sore throat, fatigue, headache and conjunctivitis may also occur [5-7]. The number of confirmed COVID-19 cases in children and

\section{ADDRESS FOR CORRESPONDENCE:}

Małgorzata Wieteska, Department of Epidemiology and Clinical Research Methodology, Medical University

of Lublin, Radziwiłłowska 11, 20-080 Lublin, Poland, e-mail: malgosia.wieteska@onet.eu 
adolescents remains underestimated, because of asymptomatic and mildly symptomatic patients. They are often not referred for COVID-19 testing as a result of availability and targeted strategies of current testing methods. Serological investigations, as well as molecular tests (RT-PCR) tend to not include pediatric patients. As a consequence, pediatric COVID-19 cases contribute to 2.1-7.8\% of all confirmed cases according to the national statistics from Asian, European and North American countries [8]. COVID-19 has initially been described as a disease that affects children less than adults due to its milder clinical manifestations. Despite this fact, a small number of patients have developed systemic hyperinflammatory syndrome, which is defined by The Royal College of Paediatrics and Child Health (RCPCH) as a pediatric inflammatory multisystem syndrome temporally associated with COVID-19 (PIMS-TS). World Health Organization (WHO) and Centers for Disease Control and Prevention (CDC) referred to this condition as a multisystem inflammatory syndrome in children (MIS-C). PIMS-TS is a systemic disorder temporally associated with severe acute respiratory syndrome coronavirus 2 (SARS-CoV-2) $[9,10]$. The first case of PIMS-TS was identified in April, 2020 [11]. However, the first patient with PIMS-TS in Poland was diagnosed in May, 2020 [12]. According to the definition, diagnostic criteria include temporal association with COVID-19, the features of systemic inflammatory response (fever, laboratory markers of inflammation, multi-organ affect) and no other diagnosis found [13]. Symptoms in Kawasaki disease (KD), toxic shock syndrome (TSS), bacterial sepsis and macrophage activation syndrome (MAS) may resemble the features of PIMSTS [10]. The aim of this work is to systematize available knowledge and conduct a review of the latest research on PIMS-TS, its epidemiology, pathogenesis, symptoms, diagnosis, treatment and differentiation. The information used in the presented analysis was obtained from the newest clinical practice guidelines and academic research databases: Google Scholar and PubMed.

\section{STATE OF KNOWLEDGE}

\section{EPIDEMIOLOGY AND PATHOGENESIS}

Data submitted so far by clinicians are still insufficient to completely understand the disease. Clinical presentation and characteristics of patients with PIMS-TS remain heterogenous. The collected research results indicate that there is a direct correlation between PIMS-TS and SARSCoV-2, however the exact mechanism of disease development remains unknown. A significant increase in the number of cases has been observed in locations that have been hardest hit by the pandemic, such as Italy, the UK, and New York City. Moreover, the vast majority of children with PIMS-TS (87\%) have had a positive serologic test result for SARS-CoV-2. That suggests a post-infectious induction of the immune response, as a result of recent coronavirus infection. Early manifestation of COVID-19 may be demonstrated by several symptoms and signs or be completely unobservable in child population. The pulmonary phase of SARS-CoV-2 infection in children is much milder or even absent, in contrast to the adult population, where it is often more severe. It is caused by reduced gene expression of the angiotensin-converting enzyme-2. Early stages of infection cause macrophage activation and then stimulation of helper T-cells. This leads to massive cytokine release, stimulation of macrophages, neutrophils and monocytes (as a result of the cytokine storm), but also leads to activation of B-cells and plasma cells responsible for antibody production. This mechanism increases the hyperimmune response, which lays at the basis of PIMS-TS pathogenesis [14]. According to data from the US, it can be concluded that the prevalence of PIMS-TS is estimated to be at 1 case per 1000 children infected by SARS-CoV-2. Due to latest research, the median age of patients suffering from PIMS-TS is 9 years, whilst mortality reaches about 1.5 to $2 \%$ among hospitalized patients $[15,16]$. The mechanisms of pathogenesis of PIMS-TS have not yet been clearly established, but an autoimmune etiology is presumable [17]. An abnormal immune response, also known as a cytokine storm is believed to be the crucial cause for the development of PIMS-TS. This reaction is characterized by high levels of inflammatory cytokines such as IL-6, IL-1b or IL-10, which contribute the most to the exaggerated immune response. This may lead to macrophage activation syndrome and multisystem organ failure. Other hypotheses have suggested that PIMS-TS is related to a delayed cytokine storm response due to inhibition of the interferon type I and III reaction. Recent research has suggested that elevated IL-6 is considered to be the most important factor associated with a cytokine storm. Elevated IL-6 is also correlated with a worse prognosis $[13,18]$.

\section{SYMPTOMS AND DIAGNOSIS}

Regarding the severity of disease, the clinical profile of patients suffering from PIMS-TS is heterogenous. The course of the PIMS-TS may be both mild (where the patient is hemodynamically stable) and severe (in patients with decompensated shock who require invasive mechanic ventilation and mechanical circulation support) [19]. In accordance with the guidelines of an expert group at the Polish Paediatric Society and the National Consultant in the field of pediatrics, fever (usually over $38.5^{\circ} \mathrm{C}$ ) is a sign that must present for at least 3 days in order for a diagnosis of PIMS-TS to be made. Laboratory investigations are also obligatory in order to confirm the diagnosis: very high levels of inflammatory markers such as C-reactive protein, procalcitonin, erythrocyte sedimentation rate, fibrinogen, D-dimer, lactate dehydrogenase and ferritin. Another criterion for the diagnosis of PIMS-TS is the presence of multiple organ damage that develop 
symptoms from at least two organs or organ systems. The disease may affect: gastrointestinal tract, circulation system, nervous system, urinary system, respiratory system, skin and mucous membranes (Table 1). According to the data collected under the auspices of the National Consultant of Pediatrics, the most frequent features of PIMS-TS in Poland include dermatologic symptoms (78\% of patients), gastrointestinal symptoms (78\% of patients), mucocutaneous symptoms (56\% of patients), neurologic symptoms (56\% of patients), musculoskeletal symptoms (56\% of patients) and respiratory symptoms (44\% of patients). Other differential diagnoses that should be excluded in order to confirm a case of PIMS-TS include infectious diseases, sepsis, toxins, TSS, hemophagocytic lymphohistiocytosis (HLH)/MAS, acute viral infection, acute appendicitis and peritonitis, connective tissue disease or inflammatory bowel disease [20,21]. There is a potential risk for coronary artery abnormalities development in approximately $25 \%$ of patients. Moreover coronary artery aneurysms are the most frequent complication of PIMS-TS. Therefore performance of an echocardiography examination is necessary as soon as the final diagnosis is made [21]. Other typical symptoms of PIMS-TS include: tachycardia, hypotension, neutrophilia, lymphopenia, as well as elevated T-troponin and B-type natriuretic peptide level [22]. According to research carried out in the USA, the most common symptoms of PIMS-TS are: fever (100\% of patients), abdominal pain (53\% of patients), skin rash (52\% of patients), and conjunctivitis ( $45 \%$ of patients). Moreover, up to $50 \%$ of patients suffered from circulatory shock and so treatment in an intensive care unit (ICU) was necessary [15]. By contrast, the Polish findings report patients predominantly present with fever ( $100 \%$ of patients), erythema or swelling of the hands and feet (78\% of patients), skin rash (67\% of patients) and also nausea, vomiting, abdominal pain, diarrhea or headache (56\% of patients). $11 \%$ of patients in Poland required intensive care treatment [20]. Among patients with diagnosed PIMS-TS with severe course of the disease in the United Kingdom, who needed intensive medical care, boys and also children from ethnic minorities (Afro-Caribbean and Hispanic ethnicity) were the most common population of patients. In Poland, in which society is homogenous and with small number of minorities, all reported patients were of Caucasian race $[20,23,24]$. The prognosis of the disorder is rather good and short-term outcome favorable, with patient survival above 95\% [25]. Intensive care admission is often necessary with median duration of 4-7 days. Despite the fact that most patients present with a severe course of PIMSTS, the case-fatality rate is estimated to be up to $2 \%$ [26].

\section{TREATMENT}

The main aim of treatment is to reduce systemic inflammation and restore organ function to decrease mortality rate and minimize the risk of severe complications
TABLE 1. Symptoms of pediatric inflammatory multisystem syndrome temporally associated with COVID-19 (PIMS-TS)

\begin{tabular}{|l|c|}
\hline Affected organ systems & Symptoms \\
\hline Gastrointestinal tract & $\begin{array}{c}\text { Severe abdominal pain } \\
\text { Vomiting } \\
\text { Diarrhea }\end{array}$ \\
\hline Circulation system & $\begin{array}{c}\text { Hypotension } \\
\text { Shock } \\
\text { Signs of myocarditis } \\
\text { Coronary artery aneurysms } \\
\text { Fluid in cardiac sac } \\
\text { Heart arrythmias }\end{array}$ \\
\hline Nervous system & $\begin{array}{c}\text { Apathy } \\
\text { Irritability }\end{array}$ \\
& $\begin{array}{c}\text { Signs of aseptic meningitis } \\
\text { Severe headache } \\
\text { Paresis or paralysis of periphe- } \\
\text { ral nerves }\end{array}$ \\
\hline Respiratory system & $\begin{array}{c}\text { Cough } \\
\text { Dyspnea }\end{array}$ \\
& $\begin{array}{c}\text { Signs of pneumonia } \\
\text { Chest pain }\end{array}$ \\
& Fluid in pleural cavity \\
\hline Urinary system & $\begin{array}{c}\text { Polymorphic skin rash } \\
\text { Conjunctivitis } \\
\text { Chapped lips }\end{array}$ \\
& $\begin{array}{c}\text { Anuria } \\
\text { Swin and mucous membranes }\end{array}$ \\
& Signs of acute kidney failure \\
\hline
\end{tabular}

of MIS-C, such as persistent cardiac dysfunction [14]. If PIMS-TS is suspected or diagnosed, a multidisciplinary diagnostic and therapeutic process should be undertaken with the participation of many specialists representing different types of units: pediatric infectious diseases, cardiology, immunology, rheumatology and ICU [8]. First-line drug therapy in patients with PIMS-TS is intravenous immunoglobulin (IVIG) at the dose of $2 \mathrm{~g} / \mathrm{kg}$ (calculated using body weight), which can be administered in a single or divided dose depending on clinical features and the ejection fraction. If there is no response, a second dose of IVIG is not recommended, excluding patients with the clinical features of $\mathrm{KD}$, because of the risk of volume overload and hemolytic anemia [21, 24]. If the general condition of the child then persists or worsens, the second line treatment is an intravenous administration of methylprednisolone at the dose of $2 \times$ $1 \mathrm{mg} / \mathrm{kg} / \mathrm{d}$. High-dose methylprednisolone is indicated for the treatment of severe cases. Other indications for the usage of glucocorticoids are: children without a therapeutic response to IVIG (24-36 h after infusion), features of shock, patients age of $<12$ months, presence of aneurysms in coronary arteries demonstrated in echocardiography and an anaphylactic reaction to IVIG. The dose should be calculated using ideal body weight, which matters particularly in overweight or obese children. Pulse 
dosing of methylprednisolone (maximum daily dose $1000 \mathrm{mg}$ ) may be considered for 3-5 days in patients with signs of shock and in deterioration of the general condition [21]. Biological therapy in children who do not respond to the previous treatment may include: anakinra (interleukin 1 receptor antagonist), tocilizumab (interleukin-6 receptor antagonist) or infliximab (tumor necrosis factor- $\alpha$ antagonist). Patients diagnosed with PIMS-TS who are SARS-CoV-2 positive on testing should also be considered for additional antiviral therapy. Remdesivir is a broad-spectrum antiviral medication that can be used in the treatment of COVID-19 in children with diagnosed pneumonia and requiring oxygen therapy. A 5-day remdesivir regimen should be given to patients with oxygen saturation $<94 \%$ who had been symptomatic for less than 10 days before receiving the first dose of remdesivir. That treatment plan is approved by the Food and Drug Administration (FDA) for children aged $\geq 12$ years and weighing $\geq 40 \mathrm{~kg}$ or in lifesaving treatment of hospitalized pediatric patients aged $<12$ years and weighing $\geq 3,5 \mathrm{~kg}$ $[21,27,28]$. Routine antibiotic treatment in children with PIMS-TS is not recommended. The administration of intravenous antibiotics in accordance with the clinical presentation of the patient is reasonable only if the diagnosis is uncertain, especially in severe cases. Additionally, children with PIMS-TS presenting clinical features of KD should receive acetylsalicylic acid (ASA) at the dose of $30-50 \mathrm{mg} / \mathrm{kg} / \mathrm{d}$ in four divided doses. After 48 hours from resolution of fever it should be reduced to $3-5 \mathrm{mg} /$ $\mathrm{kg} / \mathrm{d}$ in a single dose. Also among patients taking glucocorticoids there is a need to reduce the dose. In other cases patients should receive ASA at the dose of 3-5 mg/kg/d (the maximum daily dose of ASA must not exceed $75 \mathrm{mg}$ ) at least for 6 weeks until coronary artery abnormalities are excluded in follow-up echocardiography [11, 21]. PIMS-TS is associated with many complications such as cardiac dysfunction or acute cardiac decompensation, which can lead to the clinical manifestations of cardiogenic shock $[29,30]$. If signs of shock or hypotension are presented, fluid replacement by volume expansion using crystalloids may be useful. Vasopressors cause the constriction of blood vessels, which leads to a rise in blood pressure. This mechanism is important in the treatment of fluid resistant hypotension in children with PIMS-TS. First-line therapy includes epinephrine, while persistent shock requires the administration of norepinephrine. Usage of inotropes such as dopamine, milrinone or levosimendan may be also considered. Because of the risk of fluid overload in patients suffering from severe myocardial dysfunction, caution is required in fluid and electrolyte therapy $[8,25]$. The use of invasive mechanical ventilation and mechanical circulatory support may be necessary in severe cases [19]. Ambulatory care remains sufficient in patients who are in a good medical state and do not fulfil the criteria of PIMS-TS, but whose clinical presentation suggests a high probability of the diagnostic confirma- tion in the near future. This group of children should be supervised by a doctor due to a high risk of developing symptomatic PIMS-TS. Blood pressure measurement is essential in early detection of MIS-C (also in presumably mild cases) [21, 31]. Because of the lack of evidence, current therapeutic strategies are empirical. They are focused on symptomatic and immune-modulating treatment. In the future, there is a need to develop appropriate guidelines based on complete and multidisciplinary follow-up. It would allow for more effective evidence-based treatment of PIMS-TS [28].

\section{DIFFERENTIATION}

PIMS-TS is a recently described disorder, which shares several similarities to well-known and defined disorders such as KD, TSS or MAS. Because of that a differential diagnosis in order to make a clear diagnosis is necessary. As opposed to PIMS-TS, KD occurs seasonally, develops in a younger population (mostly children up to 5 years old), presents with longer duration of fever and a lack of gastrointestinal symptoms [23]. Patients diagnosed with PIMS-TS are more prone to cardiac arrythmias and hypotension in comparison to patients suffering from KD. They also suffer from lymphopenia, however in patients with KD lymphocytosis is characteristic [14]. An increased level of neutrophils and a decreased level of platelets is observed in PIMS-TS when compared to patients with $\mathrm{KD}$, as well as higher levels of inflammatory markers (C-reactive protein and procalcitonin) [13, 32]. A distinguishing feature is also an increased level of cardiac injury biomarkers such as B-type natriuretic peptide or troponin in patients suffering from PIMS-TS [16]. Asian children are especially prone to the development of $\mathrm{KD}$, while increased incidence of PIMS-TS among this group of the population was not observed. The similarity in signs and symptoms, and lack of specific diagnostic tests for KD or PIMS-TS make it difficult to make a final diagnosis. Etiology of severe inflammatory syndrome in children can only be determined by testing for antiSARS-CoV-2 antibodies. A positive test result indicates previous infection which is related to the pathogenesis of PIMS-TS $[33,34]$. The differences between symptoms of both disease entities result from their different immunology. KD affects medium-sized blood vessels, however PIMS-TS is characterized by multi-organ damage involving the bowel, cardiac muscle or the brain [17]. Another disease entity that should be included in the differential diagnosis of PIMS-TS is TSS. TSS is caused by the toxins produced by bacteria such as Staphylococcus aureus or Streptococcus pyogenes. Its pathogenesis leads to uncontrolled, excessive activation of the immune system caused by superantigens [14]. Differential diagnosis of PIMS-TS and TSS is based on microbiological investigations. Confirmation of the presence of staphylococci or streptococci in a microbiological culture indicates TSS [35]. MAS 
is a secondary form of HLH, characterized by a strong immune response which is induced by a cytokine storm. An excessive number of immune cells, including macrophages, leads to multi-organ failure. Its symptoms include high fever, alongside an elevated level of C-reactive protein, triglycerides and D-dimers. MAS may lead to cardiac dysfunction, central nervous system dysfunction or liver failure. The fibrinogen level in HLH/MAS is lower than the reference range and it is elevated in PIMS-TS $[21,36,37]$. To make a diagnosis of HLH there is a need to conduct molecular investigations to detect genetic mutations, or confirm 5 out of 8 of diagnostic criteria (which include: fever, splenomegaly, cytopenia, hypertriglyceridemia or hypofibrinogenemia, increased level of ferritin, decreased or absence of NK-cell activity, hemophagocytosis in the bone marrow, spleen or lymphatic nodes, and elevated concentration of soluble interleukin 2 receptor). When suspecting PIMS-TS it is crucial both to compare the clinical signs and symptoms of these conditions to make a proper diagnosis [14].

\section{SUMMARY}

Initial reports stated that children were considered to be less prone to COVID-19 than adults. On April 27, 2020, the National Health Service in the United Kingdom published a warning about pediatric patients with a severe Kawasaki-like syndrome, which has been termed as PIMS-TS [38]. Since then, thousands of new cases of this disease have been diagnosed globally. PIMS-TS is a new disorder that belongs to the group of inflammatory diseases diagnosed in children [13]. It is characterized by a wide range and diverse of symptoms, coming from several organs and organ systems [15, 20-22]. Many patients present with a relatively severe clinical status. Nevertheless, the prognosis of the patients is good $[15,20,25,26]$. The basic clinical care for these patients include multidisciplinary cooperation between specialists and symptomatic treatment until the exact pathogenesis of PIMS-TS is understood [13]. Worsening of the clinical status in patients suffering from PIMS-TS may lead to hospitalization in the ICU $[15,26]$. In order to confirm the existing hypotheses, larger cohorts within prospective trials and international collaboration are needed [28]. Because of many asymptomatic and mild cases of COVID-19, the underestimated number of diagnosed pediatric patients is alarming. There is a need to increase the number of RT-PCR tests in child population [7, 39]. Detection of patients at a high risk of developing MIS-C would allow them to receive earlier medical support and to reduce the severity of PIMS-TS [7]. Reviews of various names of the disorder, including MIS-C or PIMS-TS, should promote one definition to be as precise as possible [26]. Research in the nearest future should focus on a comprehensive understanding of the immunology of PIMS-TS, and therefore on developing uniform guidelines associat- ed with accurate treatment in order to alleviate the course of the disease and to limit the number of complications.

\section{DISCLOSURE}

The authors declare no conflict of interest.

\section{REFERENCES}

1. Esakandari H, Nabi-Afjadi M, Fakkari-Afjadi J, et al. A comprehensive review of COVID-19 characteristics. Biol Proced Online 2020; 22: 19.

2. Liu XF, Xu XK, Wu Y. Mobility, exposure, and epidemiological timelines of COVID-19 infections in China outside Hubei province. Sci Data 2021; 8: 54.

3. Asselah, T, Durantel D, Pasmant E, et al. COVID-19: Discovery, diagnostics and drug development. J Hepatol 2021; 74: 168-184.

4. Dasgupta A, Kalhan A, Kalra S. Long term complications and rehabilitation of COVID-19 patients. J Pak Med Assoc 2020; 70: 131-135.

5. Pascarella G, Strumia A, Piliego C, et al. COVID-19 diagnosis and management: a comprehensive review. J Intern Med 2020; 288: 192-206.

6. Patel NA. Pediatric COVID-19: Systematic review of the literature. Am J Otolaryngol 2020; 41: 102573.

7. Parri N, Lenge M, Buonsenso D, et al. Children with Covid-19 in Pediatric Emergency Departments in Italy. N Engl J Med 2020; 383 : 187-190.

8. Jiang L, Tang K, Levin M, et al. COVID-19 and multisystem inflammatory syndrome in children and adolescents. Lancet Infect Dis 2020; 20: e276-e288.

9. Lawrensia S, Henrina J, Wijaya E, et al. Pediatric Inflammatory Multisystem Syndrome Temporally Associated with SARS-CoV-2: a New Challenge amid the Pandemic. SN Compr Clin Med 2020; 1-9.

10. Ramcharan T, Nolan O, Lai CY, et al. Paediatric Inflammatory Multisystem Syndrome: Temporally Associated with SARS-CoV-2 (PIMS-TS): Cardiac Features, Management and Short-Term Outcomes at a UK Tertiary Paediatric Hospital. Pediatr Cardiol 2020; 41: 1391-1401.

11. Harwood R, Allin B, Jones CE, et al. A national consensus management pathway for paediatric inflammatory multisystem syndrome temporally associated with COVID-19 (PIMS-TS): results of a national Delphi process. Lancet Child Adolesc Health 2021; 5: 133-141.

12. Tracewski P, Ludwikowska K, Szenborn L, et al. The first case of pediatric inflammatory multisystem syndrome temporally associated with SARS-CoV-2 infection (PIMS-TS) in Poland, complicated by giant coronary artery aneurysms. Kardiol Pol 2020; 78 : 1064-1065.

13. Evans C, Davies P. SARS-CoV-2 paediatric inflammatory syndrome. Paediatr Child Health 2020; 10.1016/j.paed.2020.12.003.

14. Nakra NA, Blumberg DA, Herrera-Guerra A, et al.; Multi-System Inflammatory Syndrome in Children (MIS-C) Following SARS-CoV-2 Infection: Review of Clinical Presentation, Hypothetical Pathogenesis, and Proposed Management. Children (Basel) 2020; 7: 69.

15. Godfred-Cato S, Bryant B, Leung J, et al. COVID-19-Associated Multisystem Inflammatory Syndrome in Children - United States, March-July 2020. MMWR. Morb Mortal Wkly Rep 020; 69: 10741080.

16. Whittaker E, Bamford A, Kenny J, et al. Clinical Characteristics of 58 Children With a Pediatric Inflammatory Multisystem Syndrome Temporally Associated With SARS-CoV-2. JAMA 2020; 324: 259-269. 
17. Consiglio CR, Cotugno N, Sardh F, et al. The Immunology of Multisystem Inflammatory Syndrome in Children with COVID-19. Cell 2020; 183: 968-981.e7.

18. Niño-Taravilla C, Espinosa-Vielma YP, Otaola-Arca H, et al. Pediatric Inflammatory Multisystem Syndrome Temporally Associated with SARS-CoV-2 Treated with Tocilizumab. Pediatr Rep 2020; 12: 142-148.

19. Kaushik S, Aydin SI, Derespina KR, et al. Multisystem Inflammatory Syndrome in Children Associated with Severe Acute Respiratory Syndrome Coronavirus 2 Infection (MIS-C): A Multi-institutional Study from New York City. The J Pediatr 2020; 224: 24-29.

20. Okarska-Napierała M, Ludwikowska KM, Szenborn L, et al. Pediatric Inflammatory Multisystem Syndrome (PIMS) Did Occur in Poland during Months with Low COVID-19 Prevalence, Preliminary Results of a Nationwide Register. J Clin Med 2020; 9: 3386.

21. Okarska-Napierała M, Ludwikowska K, Książyk J, et al. Postępowanie z dzieckiem z wieloukładowym zespołem zapalnym powiązanym z COVID-19. Wytyczne grupy eksperckiej przy Polskim Towarzystwie Pediatrycznym i Konsultancie Krajowym w dziedzinie pediatrii. Przegląd Pediatryczny 2021; 50: 1-11.

22. Radia T, Williams N, Agrawal P, et al. Multi-system inflammatory syndrome in children \& adolescents (MIS-C): A systematic review of clinical features and presentation. Paediatr Respir Rev 2020; S1526-0542(20)30117-2.

23. Davies P, Evans C, Kanthimathinathan HK, et al. Intensive care admissions of children with paediatric inflammatory multisystem syndrome temporally associated with SARS-CoV-2 (PIMS-TS) in the UK: a multicentre observational study. Lancet Child Adolesc Health 2020; 4: 669-677.

24. Henderson LA, Canna SW, Friedman KG, et al. American College of Rheumatology Clinical Guidance for Multisystem Inflammatory Syndrome in Children Associated With SARS-CoV-2 and Hyperinflammation in Pediatric COVID-19: Version 2. Arthritis Rheumatol 2021; 73: e13-e29.

25. Fernández-Sarmiento J, De Souza D, Jabornisky R, et al. Paediatric inflammatory multisystem syndrome temporally associated with COVID-19 (PIMS-TS): a narrative review and the viewpoint of the Latin American Society of Pediatric Intensive Care (SLACIP) Sepsis Committee. BMJ Paediatr Open 2021; 5: e000894.

26. Hoste L, Van Paemel R, Haerynck F. Multisystem inflammatory syndrome in children related to COVID-19: a systematic review. Eur J Pediatr 2021; 1-16.

27. Marczyńska M, Pokorska-Śpiewak M, Talarek E, et al. Postępowanie z dzieckiem z COVID-19. Zalecenia dla pediatrów oraz lekarzy medycyny rodzinnej w podstawowej opiece zdrowotnej oraz dla leczących dzieci zakażone SARS-CoV-2 w warunkach szpitalnych. Przegl Pediatr 2020; 4: 10-16.

28. Felsenstein S, Willis E, Lythgoe H, et al. Presentation, Treatment Response and Short-Term Outcomes in Paediatric Multisystem Inflammatory Syndrome Temporally Associated with SARS-CoV-2 (PIMS-TS). J Clin Med 2020; 9: 3293.

29. Carter MJ, Shankar-Hari M, Tibby SM. Paediatric Inflammatory Multisystem Syndrome Temporally-Associated with SARS-CoV-2 Infection: An Overview. Intensive Care Med 2021; 47: 90-93.

30. Belhadjer Z, Méot M, Bajolle F, et al. Acute Heart Failure in Multisystem Inflammatory Syndrome in Children in the Context of Global SARS-CoV-2 Pandemic. Circulation 2020; 142: 429-436.

31. Dufort EM, Koumans EH, Chow EJ, et al. Multisystem Inflammatory Syndrome in Children in New York State. N Engl J Med 2020; 383: $347-358$

32. Toubiana J, Cohen JF, Brice J, et al. Distinctive Features of Kawasaki Disease Following SARS-CoV-2 Infection: a Controlled Study in Paris, France. J Clin Immunol 2021; 41: 526-535.
33. Rowley AH. Understanding SARS-CoV-2-related multisystem inflammatory syndrome in children. Nat Rev Immunol 2020; 20: 453-454.

34. Perez-Toledo M, Faustini SE, Jossi SE et al. Serology confirms SARS-CoV-2 infection in PCR-negative children presenting with Paediatric Inflammatory Multi-System Syndrome. Preprint. medRxiv 2020; 2020.06.05.20123117.

35. White M, Tiesman B, Handforth J, et al. Paediatric inflammatory multisystem syndrome temporally associated with SARS-CoV-2 (PIMS-TS): the Evelina Experience. Arch Dis Child 2020; 105 : 1025-1027.

36. Guo L, Lu MP, Dong GJ, et al. Clinical and laboratory features of macrophage activation syndrome Chin J Contemp Pediatr 2017; 19: 188-192.

37. George MR. Hemophagocytic lymphohistiocytosis: review of etiologies and management. J Blood Med 2014; 5: 69-86.

38. Diorio C, Henrickson SE, Vella LA, et al.; Multisystem inflammatory syndrome in children and COVID-19 are distinct presentations of SARS-CoV-2. J Clin Invest 2020; 130: 5967-5975.

39. Jackowska T, Marczyńska M, Peregud-Pogorzelski J. Guidance for paediatric emergency departments/rooms and departments of paediatrics on the management of a child suspected of or diagnosed with COVID-19. Pediatr Pol 2020; 95: 65-72. 